\title{
Evidence of the environmental impact of noise pollution on biodiversity: a systematic map protocol
}

Romain Sordello ${ }^{1 *}$, Frédérique Flamerie De Lachapelle ${ }^{2}$, Barbara Livoreil ${ }^{3}$ and Sylvie Vanpeene ${ }^{4}$

\begin{abstract}
Background: For decades, biodiversity has suffered massive losses worldwide. Urbanization is one of the major drivers of extinction because it leads to the physical fragmentation and loss of natural habitats and it is associated with related effects, e.g. pollution and in particular noise pollution given that many man-made sounds are generated in cities (e.g. industrial and traffic noise, etc.). However, all human activities generate sounds, even far from any human habitation (e.g. motor boats on lakes, aircraft in the air, etc.). Ecological research now deals increasingly with the effects of noise pollution on biodiversity. Many studies have shown the impacts of anthropogenic noise and concluded that it is potentially a threat to life on Earth. The present work describes a protocol to systematically map evidence of the environmental impact of noise pollution on biodiversity. The resulting map will inform on the species most studied and on the demonstrated impacts. This will be useful for further primary research by identifying knowledge gaps and in view of further analysis, such as systematic reviews.

Methods: Searches will include peer-reviewed and grey literature published in English and French. Two online databases will be searched using English terms and search consistency will be assessed with a test list. No geographical restrictions will be applied. The subject population will include all species. Exposures will include all types of man-made sounds (industrial, traffic, etc.) in all types of environments (or media) (terrestrial, aerial, aquatic), including all contexts and sound origins (spontaneous or recorded sounds, in situ or laboratory studies, etc.). All relevant outcomes will be considered (space use, reproduction, communication, abundance, etc.). An open-access database will be produced with all relevant studies selected during the three screening stages. For each study, the database will contain metadata on key variables of interest (species, types of sound, outcomes, etc.). This database will be available in conjunction with a map report describing the mapping process and the evidence base with summary figures and tables of the study characteristics.
\end{abstract}

Keywords: Noise, Pollution, Man-made sounds, Anthropogenic sounds, Masking auditory, Urban noise, Traffic noise, Impact of urbanization, Species loss, Natural habitats, Ecosystems

\section{Background}

For decades, biodiversity has suffered massive losses worldwide. Species are disappearing (e.g. [36]), populations are collapsing (e.g. [15]), species' ranges are changing (both shrinking and expanding) at unprecedented rates (e.g. [7]) and communities are being displaced by

\footnotetext{
*Correspondence: romain.sordello@mnhn.fr

1 UMS Patrimoine Naturel (PATRINAT), AFB-CNRS-MNHN, 36 rue

Geoffroy-Saint-Hilaire CP41, 75005 Paris, France

Full list of author information is available at the end of the article
}

invasive alien species (e.g. [24]). All the above are caused by human activities and scientists regularly alert the international community concerning our responsibility [30]. In particular, urban growth is one of the major reasons for biodiversity loss [21,29] in that it destroys natural habitats, fragments the remaining ecosystems (e.g. [40]) and also has other impacts, such as pollution. For example, cities produce artificial light at night that disturbs circadian rhythms, impacting plants and animals $[2,13]$. Similarly, many man-made sounds are generated in cities, by traffic and numerous human activities 
(industrial, commercial, etc.) [39]. In fact, anthropogenic noise is omnipresent and ranges beyond cities. All human activities generate noise, even far from cities (e.g. motor boats on lakes, aircraft in the sky, etc.) and those sounds can reach wild, uninhabited places [16].

Many studies have shown that such sounds may have considerable impact on animals. However, sound is not a problem in itself. A majority of species use, hear and emit sounds (e.g. Romer and Bailey 1990 [32]). Sounds are often used to communicate between partners or conspecifics, or to detect prey or predators. The problem arises when sounds turn into "noise", i.e. a disturbance or even a form of pollution. In this case, man-made sounds can mask and inhibit animal sounds and/or animal audition and it has been shown to affect communication [37], use of space [10] or reproduction [3]. This problem affects many biological groups such as birds [19], amphibians [9], reptiles [22], fish [1], mammals [34, 35] and invertebrates [6]. It spans several types of ecosystems including terrestrial [18], aquatic [17] and coastal ecosystems [33]. Many types of sounds produced by human activities would seem to be a form of noise pollution affecting biodiversity, including traffic [20], ships [38], aircraft [4] and industrial activities [23]. Noise pollution can also act in synergy with other disturbances, for example light pollution [26].

For decades, noise regulations have focused on human disorders but recently, public policies in biodiversity conservation have started to pay more attention to noise pollution. In 1996, for the first time, the European Commission's Green Paper on Future Noise Control Policy dealt with noise pollution from the point of view of environmental protection. Today in Europe, quiet areas are recommended to guarantee the tranquility of fauna [12]. Since 2000 in France, an article in "Code de l'environnement" (art. L571-1) has contained the terms "harms the environment" with respect to disturbances due to noise. To further mitigate the effects of noise pollution on biodiversity, the French Ecology Ministry wants to obtain more information on the impacts of noise on biodiversity in order to initiate policies focused on species which are known to be highly exposed. The Ministry is also interested in the types of impacts that have been effectively demonstrated and in the types of noise that have been proven to affect wildlife. We proposed to produce a systematic map of the literature dealing with this issue to provide the Ministry with a report on current knowledge and to identify sectors (sources, types of impact, etc.) where research is needed to fill in knowledge gaps.

A preliminary search did not identify any existing systematic maps or reviews, however a few reviews of the literature have been published. Most of them concern only one biological group, such as Morley et al. [25] on invertebrates, Patricelli and Blickley [27] on birds and Popper and Hastings [28] on fish. A synthesis published by Shannon et al. in 2016 [34] is more general and comes closer to a systematic map, but the search strategy would seem to be incomplete. The literature search was performed on only one database (ISI Web of Science within selected subject areas) and the review did not include grey literature. Finally, a meta-analysis was performed by Roca et al. [31], but it dealt exclusively with birds and amphibians and the authors were interested in only one effect (vocalization adjustment).

This report describes the protocol used to develop a systematic map of noise pollution and biodiversity. The systematic map will provide further information on the knowledge currently available on this issue. It will include all the relevant studies (with grey literature) collected after three screening stages. An open-access database will be produced, containing metadata for each study on key variables of interest (species, types of sound, effects, etc.). This database will be available in conjunction with a map report describing the mapping process and the evidence base. It will include aggregate data and tables of the study characteristics to highlight any gaps in the research evidence concerning the issue.

\section{Objective of the map}

The objective of the systematic map is to assess the biological and ecological impacts of noise pollution. Noise pollution is considered here as anthropogenic noise. It does not include noise made by other animals (e.g. chorus frogs) or natural events (e.g. thunder, waterfalls). The systematic map will address all man-made noise whatever its origin (road traffic, industrial machines, boats, planes, etc.), its environment or media (terrestrial, aquatic, aerial) or its type (infrasound, ultrasound, white noise, etc.). The goal is to provide a comprehensive image of the available knowledge on this topic and to quantify the literature by taxonomic groups, types of impacts and even types of studies. For this reason, the systematic map will cover all species. It will deal with all kinds of impacts, from biological to ecological (use of space, reproduction, communication, abundance, etc.). It will encompass in situ studies as well as ex situ studies (aquariums, laboratories, cages, etc.).

The primary question is: what is the evidence that manmade noise impacts biodiversity?

The secondary question is: which species, kinds of impacts and types of noise are most studied?

The components of the systematic map are detailed in Table 1. 
Table 1 Components of the systematic map

\begin{tabular}{ll}
\hline Population & All wild species \\
\hline Exposure & $\begin{array}{r}\text { All anthropogenic noises (e.g. traffic noise, urban noises, aircraft, industry, ships, etc.) in all environments and media (terrestrial, aquatic, } \\
\text { aerial), for all contexts and origins of the noise (spontaneous or recorded, in situ or in laboratory, etc.) and for all types of sound } \\
\text { (including ultrasounds, infrasounds, etc.) }\end{array}$ \\
$\begin{array}{l}\text { Conditions before and after exposure to noise (temporal comparator) or ecosystems exposed and not exposed to noise (spatial com- } \\
\text { parator) }\end{array}$ \\
$\begin{array}{l}\text { All outcomes related to the studied population, including but not restricted to biology/physiology (e.g. heart rate), use of space (e.g. } \\
\text { species distribution, individual movements), intra- and interspecific communication (e.g. song frequencies), species reproduction, } \\
\text { ecosystem composition (e.g. species richness, abundance) }\end{array}$
\end{tabular}

Methods

Searching for articles

Languages

Searches will be performed using exclusively English search terms.

Only studies published in English and in French will be included in this systematic map, due to limited resources and the languages understood by the map team. The list of search terms is presented below (see "Search string" section).

\section{Search string}

A scoping exercise was conducted on the "Web of Science Core Collection" database to build-up the search strings. Terms describing the exposure (noise pollution) and the population (biodiversity) were combined in an iterative manner until best performance was obtained. Terms describing effects (outcomes) were not included because the aim of the map is to document the available literature without any a priori restrictions on the types of effects measured in the articles.

The search string that produced the highest efficiency (number of hits compared to the test list) is presented below (see Additional file 1 for more details on the process to build the search string).

( $\mathrm{TI}=$ (noise OR sound\$) OR TS = ("masking auditory" OR "man-made noise" OR "anthropogenic noise" OR "man-made sound\$” OR "music festival\$" OR ((pollution OR transportation OR road\$ OR highway\$ OR motorway\$ OR railway\$ OR traffic OR urban OR city OR cities OR construction OR ship\$ OR boat\$ OR port\$ OR aircraft\$ OR airplane\$ OR airport\$ OR industr* OR machinery OR "gas extraction" OR mining OR drilling OR pile-driving OR "communication network\$" OR "wind farm\$" OR agric" OR farming OR military OR gun\$ OR visitor\$) AND noise $))$ AND TS $=($ ecolog* OR biodiversity OR ecosystem $\$$ OR "natural habitat\$" OR species OR vertebrate\$ OR mammal\$ OR reptile\$ OR amphibian\$ OR bird\$ OR fish* OR invertebrate\$ OR arthropod\$ OR insect\$ OR arachnid\$ OR crustacean\$ OR centipede\$)).

\section{Comprehensiveness of the search}

A test list of 65 scientific articles was established (see Additional file 2) and used to assess the comprehensiveness of the search string. The test list was composed of the three groups listed below.

1. Forty relevant scientific articles identified by the review team prior to the review.

2. Eight key articles identified using three relevant reviews:

- Brumm [5] (two articles);

- Cerema [8] (three articles);

- Dutilleux and Fontaine [11] (three articles).

3. Seventeen studies not readily accessible or indexed by the most common academic databases, submitted by subject experts contacted prior to the review ( 29 subject experts were contacted, 7 responded).

\section{Online publication databases}

We first listed the databases to which the members of our review team had access, databases that covered ecology and that guaranteed reproducibility (accessibility by researchers from all over the world, advanced search functions, etc.). The resource limitations weighing on the project did not allow us to cover more than two databases given the number of articles obtained during the scoping exercise.

On the basis of the criteria listed above, the two databases below were selected:

- "Web of Science Core Collection" on the Web of Science platform (Clarivate). See Additional file 3 for citation indexes included in the "Web of Science Core Collection" to which the review team had access via the team members' institutions. As explained above, the scoping exercise was conducted using this database. It returned 7859 articles (the search was run on the 14 December 2018 and covered SCI- 
EXPANDED, SSCI, A\&HCI, CPCI-S, CPCI-SSH, BKCI-S, BKCI-SSH, ESCI and CCR-EXPANDED, without any timespan restrictions). The search comprehensiveness value was 92\% (60 articles in the test list were referenced in the WOS CC and 55 were retrieved by the string).

- Scopus (Elsevier). The search string described above was adapted to take into account differences in search syntax. It returned 11,186 articles (a preliminary search was run on 14 December 2018, without any timespan restrictions). The comprehensiveness value was 92\% (61 articles in the test list were indexed in Scopus and 56 were retrieved by the string).

Approximately 6000 articles were listed in both databases. One of the articles not indexed in the WOS CC was indexed in Scopus and was retrieved by the search string. Consequently, combining the two databases, the global comprehensiveness value was 93\% (61 articles indexed and 57 articles retrieved by the search string). See Additional file 4 for more details on the comprehensiveness values.

\section{Internet searches}

Searches will be performed using the search engines:

- Google Scholar (https://scholar.google.com/);

- BASE (https://www.base-search.net) and/or CORE (https://core.ac.uk/).

The English search string detailed above will be used. If necessary, the search string will be modified as per the search-engine help files (when provided). To minimize bias in favor of published literature in search results provided by Google Scholar [14], searches will be performed on titles only and the first 300 hits will be screened (based on sorting by relevance of results if possible).

\section{Specialist sources}

The following French specialist organizations will be searched for relevant publications, including grey literature, using manual searches of their websites and automatic search facilities using French keywords if possible:

- Information and Documentation Center on Noise (http://www.bruit.fr);

- Document portal of the French Ecology Ministry (http://www.portail.documentation.developpementdurable.gouv.fr/);

- Document database of the General commission for sustainable development (http://temis.documentat ion.developpement-durable.gouv.fr/).

\section{Supplementary searches}

A call for literature will be conducted through a professional network to find non peer-reviewed literature, including reports published in French or in English. Specialized organizations will also be requested to amplify the call for literature using their network, their web forum or their mailing list. Social media (http://www. academia.edu, http://www.researchgate.net and http:// www.linkedin.com) will be used to alert the research community concerning the systematic map and to request that subject experts submit non peer-reviewed publications.

\section{Article screening and study eligibility criteria Screening process}

Using the predefined inclusion/exclusion criteria detailed below, article selection will proceed according to a threestage hierarchical process, i.e. first title, then abstract and finally the full text.

If there is any doubt regarding the presence of a relevant inclusion criterion or if there is insufficient information to make an informed decision, articles will be retained for assessment at a later stage. In particular, articles retained after title screening but that do not have an abstract will be immediately transferred to full-text screening. Given that titles and abstracts in grey literature do not conform to scientific standards, assessments of grey literature will proceed immediately to the full-text screening phase. Care will be taken to ensure that reviewers never screen their own articles.

The three screening stages will be conducted by two or more reviewers. To assess the consistency of the inclusion/exclusion decisions, a Kappa test will be performed. To that end, before the actual screening process, a set of articles will be randomly selected and screened by each of the reviewers independently. The operation will be repeated until reaching a Kappa value higher than 0.6. Whatever the Kappa value, disagreements will be discussed and resolved between the reviewers before beginning the screening process.

During the scoping stage conducted in the "Web of Science Core Collection", the three stages of the screening process were tested by one reviewer in order to refine the eligibility criteria. For the articles screened during the scoping stage, a second reviewer will examine the rejected articles to assess the consistency of the inclusion/exclusion decision.

\section{Eligibility criteria}

Article eligibility will be based on the list of criteria detailed in Table 2. The list of all articles will be provided, informing the inclusion/exclusion decisions at the three 
Table 2 Inclusion/exclusion criteria for the three-stage screening process

\begin{tabular}{ll}
\hline Exclusion criteria & - Population: \\
& Domestic species or populations (e.g. cats, dogs, etc.) \\
& - Exposure: \\
& Studies that deal exclusively with natural noises (e.g. chorus frog) \\
& - Context: \\
& No restrictions on biogeographical zones \\
- Population: & All wild species, including in captivity (e.g. pandas in zoos). All terrestrial, aquatic and amphibious species \\
- Exposure: & - All man-made noises whatever the environment or media (terrestrial, aquatic, aerial) and the type of sounds (infrasounds, ultra- \\
& sounds, etc.), including artificially recreated noises (e.g. recorded road noise) and even abstract noises (e.g. white noise produced \\
& by a computer) \\
- In-situ and ex situ experiments (e.g. studies in laboratories, zoological parks, aquariums, cages, etc.) & - Outcomes: \\
& All outcomes related to the included populations, including but not restricted to biology/physiology (e.g. heart rate), use of space \\
& (e.g. species distribution, individual movements), intra and interspecific communication (e.g. song frequencies), species repro- \\
& duction, ecosystem composition (e.g. species richness, abundance) \\
&
\end{tabular}

screening stages and, in case, reasons for the exclusion (see the code book in Additional file 5).

\section{Data coding strategy}

All the studies passing the three screening stages will be included in the mapping database.

\section{Coding strategy}

Each article will be coded based on the full text using keywords and expanded comments fields describing various aspects of study (see the code book in Additional file 6).

The key variables will include:

- Study description:

- Publication source (WOS research, Scopus research, Google Scholar research, etc.);

- Basic bibliographic information (authors, title, publication date, journal, DOI, etc.);

- Language (English/French);

- Publication type (journal article, book, thesis...);

- Study content (study, review, meta-analysis, other, etc.);

- Study characteristics:

- Country where the study was conducted;

- Type of population studied (species or species groups);

- Type of exposition, source of noise (e.g. urban, transportation, industrial activity, recreation, other), type of environment or media (terrestrial, aerial, aquatic), type of noise (artificial, real, recorded);
- Type of impacts, used to describe subtopics of noise pollution (e.g. space use, reproduction, communication, abundance, etc.) in relation to the outcomes;

- Information on study quality:

- Study context: in situ (field)/ex situ (laboratory, aquariums, etc.);

- Experimental (causal)/Observational (correlative) study;

- For experimental studies, the type of comparator (spatial/temporal).

As far as possible, controlled vocabulary will be employed to code the variables (e.g. publication type, dates, country, etc.), using thesaurus or ISO standards (e.g. ISO 639-1 for the language publication variable). To categorize the sources of noise and the outcomes (effects), we will use the review Shannon et al. [34] that give an example of categorization (see in this publication Table 2, page 988 about the sources of noise and Table 3, page 889 about the impacts of noise).

Each selected article will be double coded by two reviewers. If, due to resource limitations, true double coding is not possible, an a posteriori check will be carried out by a second reviewer and potential disagreements will be discussed until a consensus is reached.

\section{Study map and presentation}

Where there is more than one study found in an article, each study will be recorded as a specific entry in the database.

The database will be open access and included as an appendix to the systematic map publication. To ensure reusability and long-term preservation, the database will, 
if possible, be deposited as a.csv file in a data repository such as Zenodo.

The final systematic map will include summary figures and tables of the study characteristics. Possible knowledge gaps (un- or under-represented subtopics that warrant further primary research) and knowledge clusters (well-represented subtopics that are amenable to full synthesis by a systematic review) will be identified e.g. by cross-tabulating key meta-data variables in heat maps (e.g. biological groups and outcomes). Based on these results, recommendations will be made on priorities for future research and mitigation of noise pollution.

\section{Additional files}

Additional file 1. Search string building process.

Additional file 2. List of eligible studies identified by subject experts.

Additional file 3. Web of Science Core Collection database subscription details.

Additional file 4. Details on database indexation of the articles in the test list for the comprehensiveness calculation.

Additional file 5. Codebook of the inclusion/exclusion decisions at the three screening stages.

Additional file 6. Codebook of the systematic map database.

\section{Authors' contributions}

RS is the project scientific coordinator of the map. RS originated the idea of the systematic map, conducted the scoping stage and wrote the draft manuscript. BL assisted the team concerning methods and CEE guidelines. FF helped with the search strategy. SV brought her expertise about noise pollution public policies. All authors read and approved the final manuscript.

\section{Authors' information}

$R S, B L$ and SV are scientists. FF is an academic librarian. BL works at the French Collaboration for Environmental Evidence Center, hosted at FRB (Paris).

\section{Author details}

1 UMS Patrimoine Naturel (PATRINAT), AFB-CNRS-MNHN, 36 rue Geoffroy-Saint-Hilaire CP41, 75005 Paris, France. ${ }^{2}$ Fondation pour la Recherche sur la Biodiversité (FRB), 75005 Paris, France. ${ }^{3}$ Université de Bordeaux, 33400 Talence, France. ${ }^{4}$ Institut de recherche en sciences et technologies pour l'environnement et l'agriculture (Irstea), 13182 Aix-en-Provence, France.

\section{Acknowledgements}

RS thanks Nicola Randall for advice.

\section{Competing interests}

The authors declare that they have no competing interests.

\section{Availability of data and materials}

Data sharing is not applicable to the systematic map protocol in that no datasets were generated for this article. Datasets produced by the systematic map will be shared publicly.

\section{Consent for publication}

Not applicable.

Ethics approval and consent to participate

Not applicable.

\section{Funding}

This research is undertaken as current work of UMS Patrimoine naturel, a joint research unit funded by AFB French Biodiversity Agency, CNRS National Scientific Research Center and MNHN National Museum of Natural History, on behalf of the French Ecology Ministry.

\section{Publisher's Note}

Springer Nature remains neutral with regard to jurisdictional claims in published maps and institutional affiliations.

Received: 20 June 2018 Accepted: 11 January 2019

Published online: 12 February 2019

\section{References}

1. Amoser S, Wysocki LE, Ladich F. Noise emission during the first powerboat race in an Alpine lake and potential impact on fish communities. J Acoust Soc Am. 2004;1 16(6):3789-97. https://doi.org/10.1121/1.1808219.

2. Bennie J, Davies WT, Cruse D, Gaston KJ. Ecological effects of artificial light at night on wild plants. J Ecol. 2016;104(3):611-20. https://doi. org/10.1111/1365-2745.12551.

3. Bernath-Plaisted J, Koper N. Physical footprint of oil and gas infrastructure, not anthropogenic noise, reduces nesting success of some grassland songbirds. Biol Conserv. 2016;204(B):434-41. https://doi.org/10.1016/j. biocon.2016.11.002.

4. Brown AL. Measuring the effect of aircraft noise on sea birds. Environ Int. 1990;16(4-6):587-92. https://doi.org/10.1016/0160-4120(90)90029-6.

5. Brumm H. Anthropogenic noise: implications for conservation. Cambridge: Academic Press; 2010. p. 89-93.

6. Bunkley JP, Mcclure CJW, Kawahara AY, Francis CD, Barber JR. Anthropogenic noise changes arthropod abundances. Ecol Evol. 2017;7(9):297785. https://doi.org/10.1002/ece3.2698.

7. Ceballos G, Ehrlich PR, Dirzo R. Biological annihilation via the ongoing sixth mass extinction signaled by vertebrate population losses and declines. PNAS. 2017;114(30):6089-96. https://doi.org/10.1073/ pnas. 1704949114.

8. Cerema. Bruit urbain et faune sauvage. Synthèse bibliographique. 2007.

9. Cunnington GM, Fahrig L. Plasticity in the vocalizations of anurans in response to traffic noise. Acta Oecologica. 2010;36(5):463-70. https://doi. org/10.1016/j.actao.2010.06.002.

10. Drolet A, Dussault C, Côté SD. Simulated drilling noise affects the space use of a large terrestrial mammal. Wildl Biol. 2016;22(6):284-93. https:// doi.org/10.2981/wlb.00225.

11. Dutilleux G, Fontaine A. Bruit urbain et faune sauvage. Paris: Rapport d'études. Cerema.; 2015.

12. European Environment Agency (2014). Good practice guide on quiet areas. EEA Technical report No 4/2014. https://doi.org/10.2800/12611.

13. Gaston KJ, Bennie J, Davies TW, Hopkins J. The ecological impacts of nighttime light pollution: a mechanistic appraisal. Biol Rev. 2013;88(4):912-27. https://doi.org/10.1111/brv.12036.

14. Haddaway NR, Collins AM, Coughlin D, Kirk S. The role of google scholar in evidence reviews and its applicability to grey literature searching. PLoS ONE. 2015;10(9):e0138237. https://doi.org/10.1371/journal.pone.0138237.

15. Hallmann CA, Sorg M, Jongejans E, Siepel H, Hofland N, Schwan H. More than 75 percent decline over 27 years in total flying insect biomass in protected areas. PLOS ONE. 2017;12(10):e0185809. https://doi. org/10.1371/journal.pone.0185809.

16. Hempton G, Grossmann J. One square inch of silence: one man's quest to preserve quiet. New York City: Free Press/Simon \& Schuster; 2009.

17. Jacobsen L, Baktoft H, Jepsen N, Aarestrup K, Berg S, Skov C. Effect of boat noise and angling on lake fish behaviour. J Fish Biol. 2014;84:1768-80. https://doi.org/10.1111/jfb.12395.

18. Koper N, Leston L, Baker TM, Curry C, Rosa P. Effects of ambient noise on detectability and localization of avian songs and tones by observers in grasslands. Ecol Evol. 2016;6(1):245-55. https://doi.org/10.1002/ ece3.1847.

19. Lazerte SE, Slabbekoorn H, Otter KA. Learning to cope: vocal adjustment to urban noise is correlated with prior experience in black-capped 
chickadees. Proc R Soc B Biol Sci. 2016;283(1833). https://doi.org/10.1098/ rspb.2016.1058.

20. Lengagne T. Traffic noise affects communication behaviour in a breeding anuran, Hyla arborea. Biol Conserv. 2008;141(8):2023-31. https://doi. org/10.1016/j.biocon.2008.05.017.

21. Macdonald RI, Kareiva P, Forman RTT. The implications of current and future urbanization for global protected areas and biodiversity conservation. Biol Conserv. 2008;141(6):1695-703. https://doi.org/10.1016/j.bioco n.2008.04.025.

22. Mancera KF, Murray PJ, Lisle A, Dupont C, Faucheux F, Phillips CJC. The effects of acute exposure to mining machinery noise on the behaviour of eastern blue-tongued lizards (Tiliqua scincoides). Anim Welf. 2017;26(1):11-24. https://doi.org/10.7120/09627286.26.1.011.

23. Mason JT, Macclure CJW, Barber JR. Anthropogenic noise impairs owl hunting behavior. Biol Conserv. 2016;199:29-32. https://doi.org/10.1016/j. biocon.2016.04.009.

24. Menon M, Mohanraj R. Temporal and spatial assemblages of invasive birds occupying the urban landscape and its gradient in a southern city of India. J Asia Pac Biodivers. 2016;9(1):74-84. https://doi.org/10.1016/j. japb.2015.12.005

25. Morley EL, Jones G, Radford AN. The importance of invertebrates when considering the impacts of anthropogenic noise. Proc R Soc B. 2013;281:2013-683. https://doi.org/10.1098/rspb.2013.2683.

26. Newport J, Shorthouse DJ, Manning AD. The effects of light and noise from urban development on biodiversity: implications for protected areas in Australia. Ecol Manag Restor. 2014;15(3):204-14. https://doi. org/10.1111/emr.12120.

27. Patricelli GL, Blickley JL. Avian communication in urban noise: causes and consequences of vocal adjustment. Auk. 2006;123(3):639-49. https://doi. org/10.1642/0004-8038(2006)123\%5b639:ACIUNC\%5d2.0.CO;2.

28. Popper AN, Hastings MC. The effects of anthropogenic sources of sound on fishes. J Fish Biol. 2009;75(3):455-89. https://doi.org/10.111 1/j.1095-8649.2009.02319.x.

29. Reis E, López-Iborra GM, Pinheiro RT. Changes in bird species richness through different levels of urbanization: implications for biodiversity conservation and garden design in Central Brazil. Landsc Urban Plan. 2012;107(1):31-42. https://doi.org/10.1016/j.landurbplan.2012.04.009.

30. Ripple WJ, Wolf C, Newsome TN, Galetti M, Alamgir M, Crist E, Mahmoud MI, Laurance WF, 15,364 scientist signatories from 184 countries.
World scientists' warning to humanity: a second notice. Bioscience. 2017;67(12):1026-8. https://doi.org/10.1093/biosci/bix125.

31. Roca IT, Desrochers L, Giacomazzo M, Bertolo A, Bolduc P, Deschesnes R, Martin CA, Rainville V, Rheault G, Proulx R. Shifting song frequencies in response to anthropogenic noise: a meta-analysis on birds and anurans. Behav Ecol. 2016;27(5):1269-74. https://doi.org/10.1093/beheco/arw060.

32. Romer H, Bailey WJ. Insect hearing in the field. Comp Biochem Physiol. 1990;97A(4):443-7.

33. Samuel Y, Morreale SJ, Clark CW, Greene CH, Richmond ME. Underwater, low-frequency noise in a coastal sea turtle habitat. J Acoust Soc Am. 2005;117(3 Pt 1):1465-72.

34. Shannon G, Mckenna MF, Angeloni LM, Crooks KR, Fristrup KM, Brown E, Warner KA, Nelson MD, White C, Briggs J, Mcfarland S, Wittemyer G. A synthesis of two decades of research documenting the effects of noise on wildlife. Biol Rev. 2016;91:982-1005. https://doi.org/10.1111/brv.12207.

35. Shannon G, Crooks KR, Wittemyer G, Fristrup KM, Angeloni LM. Road noise causes earlier predator detection and flight response in a freeranging mammal. Behav Ecol. 2016;27(5):1370-5. https://doi.org/10.1093/ beheco/arw058.

36. Stuart SN, Chanson JS, Cox NA, Young BE, Rodrigues ASL, Fischman DL, Waller RW. Status and trends of amphibian declines and extinctions worldwide. Science. 2004;306(5702):1783-6. https://doi.org/10.1126/ science.1103538.

37. Sun JWC, Narins PM. Anthropogenic sounds differentially affect amphibian call rate. Biol Cons. 2005;121(3):419-27. https://doi.org/10.1016/j. biocon.2004.05.017.

38. Vasconcelos RO, Amorim MCP, Ladich F. Effects of ship noise on the detectability of communication signals in the Lusitanian toadfish. J Exp Biol. 2007;210:2104-12. https://doi.org/10.1242/jeb.004317.

39. Warren PS, Katti M, Ermann M, Brazel A. Urban bioacoustics: it's not just noise. Anim Behav. 2006;71(3):491-502. https://doi.org/10.1016/j.anbeh av.2005.07.014

40. Xu X, Xie Y, Qi K, Luo Z, Wang X. Detecting the response of bird communities and biodiversity to habitat loss and fragmentation due to urbanization. Sci Total Environ. 2018;624(15):1561-76. https://doi.org/10.1016/j. scitotenv.2017.12.143.
Ready to submit your research? Choose BMC and benefit from:

- fast, convenient online submission

- thorough peer review by experienced researchers in your field

- rapid publication on acceptance

- support for research data, including large and complex data types

- gold Open Access which fosters wider collaboration and increased citations

- maximum visibility for your research: over $100 \mathrm{M}$ website views per year

At BMC, research is always in progress.

Learn more biomedcentral.com/submissions 